\title{
Multilagenbasierte Lab-on-a-Chip Systeme - Online Überwachung und automatisiertes Handling
}

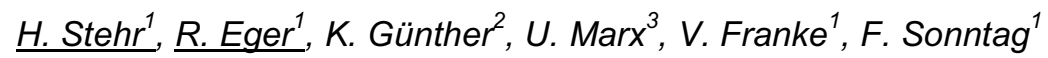 \\ ${ }^{1}$ Fraunhofer-Institut für Werkstoff- und Strahltechnik IWS, Dresden, Deutschland \\ ${ }^{2}$ Hochschule Mittweida, Fakultät MNI, Mittweida, Deutschland \\ ${ }^{3}$ Technische Universität Berlin, Institut für Biotechnologie, Berlin, Deutschland
}

\begin{abstract}
Abstrakt:
Die vorliegende Arbeit beschreibt eine geschlossene Technologiekette für die schelle, flexible und preiswerte Herstellung von Lab-on-a-Chip-Systemen als Multilagenaufbau lasermikrostrukturierter Folien sowie einen Ansatz zur Sensitivitätssteigerung für die Online Fluoreszenz-Überwachung durch Integration funktionaler Reflexionsschichten. Aufbauend auf dynamischen Multi-Organ-Chips für die Substanztestung an langzeitkultivierten humanen 3D-Co-Kulturen wird ein Multilagen-DemonstratorSystem vorgestellt und dessen Funktionalität am Beispiel der Substanztestung demonstriert. Weiterhin erfolgt die Vorstellung erster Ergebnisse zur Eignung von ta-C-Schichten als Reflektor.
\end{abstract}

Schlüsselwörter: Lab-on-a-Chip, Multilagensystem, Laser, Fluoreszenz, Kohlenstoffschichten, taC, PLD

\section{Einleitung}

Miniaturisierung, schnelle Prototypenfertigung und Automatisierung spielen im Bereich Labon-a-Chip eine zunehmend wichtigere Rolle. Lab-on-a-Chip-Systeme werden neben der Umweltanalytik und der medizinischen Diagnostik bisher vor allem als Ersatz für Tierversuche in der pharmazeutischen und kosmetischen Substanztestung angewandt.

In den letzten Jahren entwickelten die TU Berlin und das Fraunhofer IWS Dresden in enger Kooperation eine Lab-on-a-Chip-Plattform. Diese vereint verschiedene Mikropumpen, Ventile, Kanäle, Reservoire und maßgeschneiderte Zellkulturbereiche [1, 2, 3]. Diese Plattform hat das Potential die Substanztestung in der pharmazeutischen und kosmetischen Industrie $\mathrm{zu}$ revolutionieren, indem Tierversuche in den nächsten Jahren ersetzt werden könnten [4].

Bisher bilden gegossene Flusszellen aus Silikon das Herzstück der Lab-on-ChipPlattform, wobei die Mikrofluidikstrukturen im Wesentlichen in nur einer Ebene ausgeprägt sind. Für eine erfolgreiche ADMET-Testung muss die Plattform weiterentwickelt werden, damit mehrere fluidische Kreisläufe (Blutkreislaufsystem, Verdauungssystem, Ausscheidungssystem) in unterschiedlichen Ebenen realisiert werden können [3].

Weiterhin muss die Sensitivität der Online Überwachungssysteme erhöht werden, da beispielsweise bei der Vitalfärbung so wenig wie möglich Farbstoff eingesetzt werden soll.

\section{Material und Methode}

\subsection{Multilagenbasierte Lab-on-a-Chip-} Systeme

Für die schelle, flexible und preiswerte Herstellung von Lab-on-a-Chip-Systemen wurde am Fraunhofer IWS Dresden eine geschlossene Technologiekette für den Multilagenaufbau lasermikrostrukturierter Folien entwickelt und erfolgreich etabliert. Im ersten Schritt wird das zu fertigende Mikrofluidiksystem konstruktiv in einzelne Lagen zerlegt, die später jeweils durch eine separate Folie ausgebildet werden. Ausgehend von den funktionellen Randbedingungen für jede Lage, erfolgt in einem zweiten Schritt die Auswahl der entsprechenden Folien mit den notwendigen Eigenschaften (hydrophil, hydrophob, transparent, permeabel, porös). Im dritten Schritt werden die Folien mittels Lasermikromaterialbearbeitung beidseitig strukturiert und funktionalisiert. Das Zusammenfügen der einzelnen Folien zu einem Mehrlagensystem erfolgt durch Verkleben oder Plasmabonden im vierten und damit letzten Schritt. Die Multilagentechnologie ermöglicht auch die Realisierung von pneumatisch angetriebenen Mikropumpen und Ventilen, siehe Abbildung 1. 


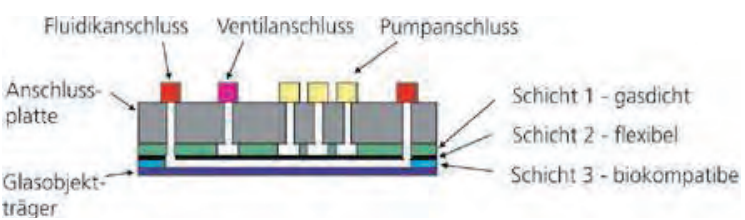

Abb. 1. Schematischer Aufbau eines Multilagen-Labon-a-Chip-Systems mit integrierten Mikropumpen und Ventilen.

Durch den Multilagenansatz können die mikrofluidischen Strukturen auf mehrere Ebenen verteilt werden, siehe Abbildung 2.

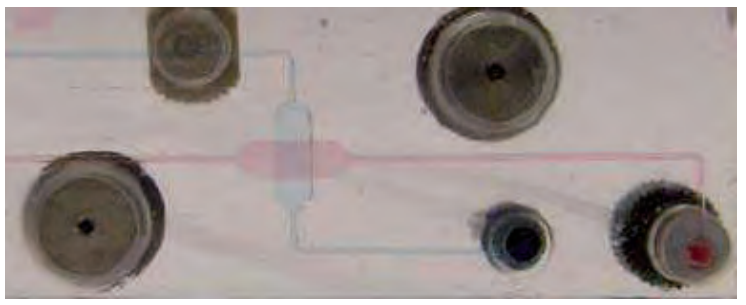

Abb. 2. Prototyp mit in verschiedenen Ebenen verlaufenden, sich kreuzenden Kanälen. Die Kanäle wurden mit zwei verschiedenfarbigen Lösungen befüllt.

Daraus resultiert eine Erhöhung der Funktionalität pro Chipfläche. Durch den Einsatz von Folien mit unterschiedlichen Eigenschaften können die Benetzung gezielt gesteuert und Funktionen wie Kapillar-StoppVentile, Barrieren oder eine gezielte Zellbesiedlung realisiert werden. Weiterhin lassen sich auch Folien mit aufgebrachten Dünnschichtelektroden integrieren, was den Einsatz elektrischer und elektrochemischer Sensoren und Aktoren ermöglicht.

\subsection{Online Fluoreszenz-Überwachung}

Für die fluoreszenzbasierte Online Überwachung wurde das von Fraunhofer IOF Jena und Fraunhofer IWS Dresden gemeinsam entwickelte Sensormodul verwendet, welches ohne mechanisch bewegte Teile die Fluoreszenzanalyse bei zwei Anregungswellenlängen ermöglicht [5]. Dabei wird das Licht zweier unterschiedlich farbiger Hochleistungsleuchtdioden überlagert und in einer Mikroskopähnlichen Anordnung auf die Probe abgebildet. Die spektrale Charakteristik wird durch die Wahl von Lichtquelle und Filtersatz bestimmt. Mit diesem System ist es möglich, durch sequenzielle Beleuchtung, d. h. Schalten der beiden unterschiedlichen Leuchtdioden, die Fluoreszenz zweier verschiedener Farbstoffe zu bestimmen. Da die Fluoreszenz in erster Näherung isotrop in alle Raumrichtungen emittiert wird, muss ein empfindliches System einen großen Raumwinkelbereich erfassen. Diese Forderung entspricht einer großen numerischen Apertur des optischen Systems, weshalb als Frontlinse eine asphärische Linse (NA 0.5) eingesetzt wird. Die optische Abbildung ist so dimensioniert, dass Kanäle mit etwa 0,5 $\mathrm{mm}$ Breite ausgeleuchtet werden.

2.3 Integrierte, funktionale Reflexionsschichten

Für eine sensitive Fluoreszenzmessung sollte an der Oberseite des zu vermessenden Volumens ein Reflektor ausgebildet sein, der nach oben emittierte Fluoreszenzstrahlung zum Detektor reflektiert, siehe Abbildung 3.

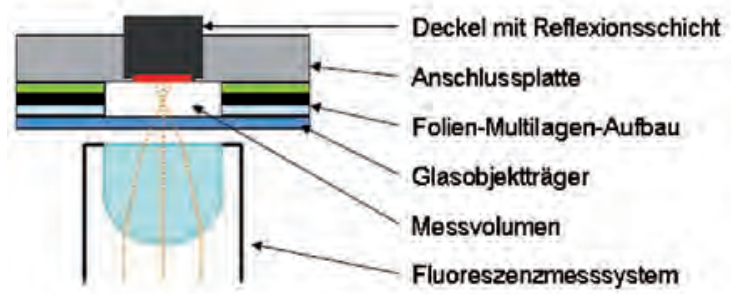

Abb. 3. Schematischer Aufbau der Fluoreszenzmessung mit integrierter, funktionaler Reflexionsschicht.

Dieser Reflektor sollte folgende Eigenschaften aufweisen:

> hohe Biokompatibilität,

$>$ geringe Eigenfluoreszenz bei den verwendeten Anregungswellenlängen

$>$ niedrige Reflexion der verwendeten Anregungswellenlängen

$>$ hohe Reflexion der entstehenden Emissionswellenlängen.

\subsection{Automatisiertes Handling}

Das automatisierte Handling der Lab-on-ChipSysteme erfolgt mit der von der GeSiM mbH und dem Fraunhofer IWS gemeinsam entwickelten, universellen Laborautomationsplattform $[6,7]$. Die Verknüpfung von FluoreszenzSensormodul und Laborautomationsplattform ermöglicht die vollautomatisierte, ortsaufgelöste Fluoreszenzmessung. Diese wird beispielsweise genutzt, um die Zellkulturkammern abzuscannen und so eine Aussage über das komplette Gewebe zu erhalten.

\section{Ergebnisse}

\subsection{Multilagen-Demonstrator-System}

Aufbauend auf gemeinsam mit der TU Berlin entwickelten dynamischen Multi-Organ-Chips für die Substanztestung an langzeitkultivierten humanen 3D-Co-Kulturen [3] wurde ein Multilagen-Demonstrator-System entworfen und erfolgreich umgesetzt. 


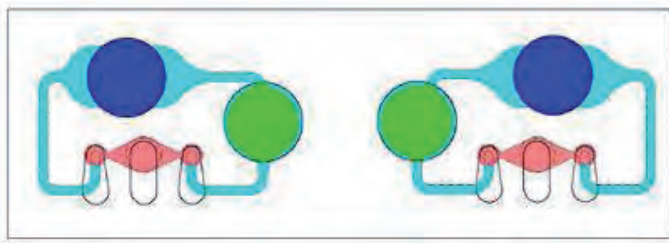

Abb. 4. Schematischer Grundaufbau des MultilagenDemonstrator-Systems, einfache Zellkulturkammer (grün), Zellkulturkammer mit integrierter Membran (blau), 3-Punkt-Peristaltikpumpe (pink).

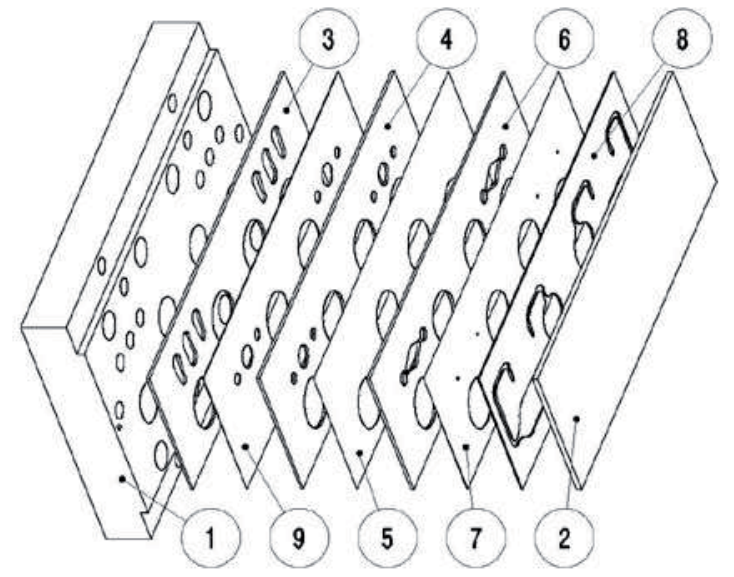

1 Anschlussplatte aus Polycarbonat mit fluischen Schnittstellen $\rightarrow$ fluidische Schnittstelle

2 Standard Glasobjektträger $\rightarrow$ optische Schnittstelle

3 Polypropylen-Folie, $200 \mu \mathrm{m}$ dick, laserstrukturiert, doppelseitig mit Klebstoff beschichtet

4 Polypropylen-Folie, $200 \mu \mathrm{m}$ dick, laserstrukturiert, doppelseitig mit Klebstoff beschichtet

5 Thermoplastische Elastomer-Folie auf Polyurethan-Basis, $25 \mu \mathrm{m}$ dick, laserstrukturiert $\rightarrow$ Pumpmembran

6 Polypropylen-Folie, $200 \mu \mathrm{m}$ dick, laserstrukturiert, doppelseitig mit Klebstoff beschichtet

7 Cycloolefin-Copolymer-Folie, $90 \mu \mathrm{m}$ dick, laserstrukturiert

8 Polypropylen-Folie, $200 \mu \mathrm{m}$ dick, laserstrukturiert, doppelseitig mit Klebstoff beschichtet

9 Mikromembran PALL Supor®-800 $0,8 \mu \mathrm{m}, 50 \mu \mathrm{m}$ dick, laserstrukturiert $\rightarrow$ Membran für Zellbesiedlung (optional)

Abb. 5. Schematische Explosionsdarstellung des Multilagen-Demonstrator-Systems.
Das System umfasst zwei geschlossene Kreisläufe, siehe Abbildung 4, bestehend aus einer einfachen Zellkulturkammer (grün), einer zweiten Zellkulturkammer (blau), die optional mit einer integrierten Membran ausgestattet werden kann, sowie einer 3-Punkt-Peristaltikpumpe (pink) [8].

Das Multilagen-Demonstrator-System setzt sich, wie in Abbildung 5 dargestellt, aus einem Stapel von sechs optional sieben, mittels Laser strukturierten Polymerfolien (3-9) zusammen. Der Stapel wird auf einer Seite durch eine Anschlussplatte (1) und auf der anderen Seite durch einen Glasobjektträger (2) abgeschlossen.

Die Strukturierung der Folien mit einer Außenabmessung von $26 \times 76 \mathrm{~mm}^{2}$ erfolgte mit einem Scanner-basierten Lasermikromaterialbearbeitungssystem. Der eingesetzte Laser hat eine Wellenlänge von $355 \mathrm{~nm}$ und eine Pulsdauer von $10 \mathrm{ps}$.

Im Anschluss an die Fertigung wurde die Funktionalität der Prototypen (Dichtigkeit, Pumpfunktion), siehe Abbildung 6, erfolgreich getestet.

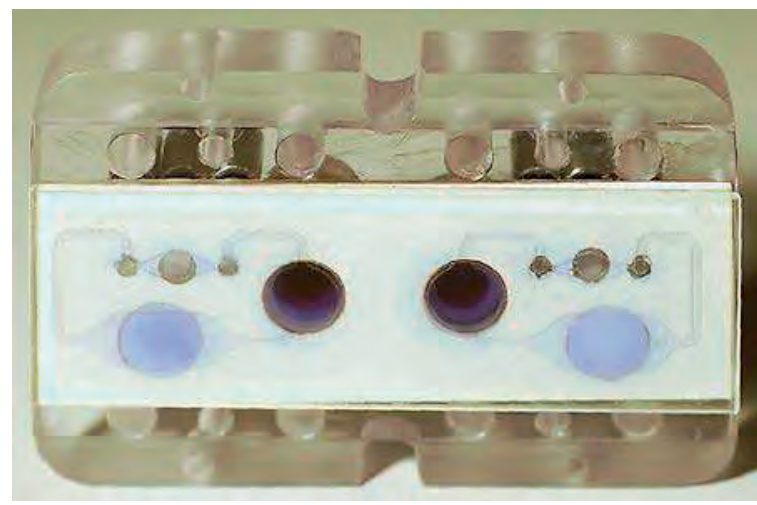

Abb. 6. Prototyp des Multilagen-DemonstratorSystems mit Farbstoff gefüllt.

\subsection{Funktionsnachweis anhand Substanz- testungs-Assay}

Der Funktionsnachweis des MultilagenDemonstrator-Systems erfolgte über die erfolgreiche Durchführung von Substantztestungs-Assays auf Basis des am Fraunhofer IWS etablierten Protokolls zur automatisierten Vitalfärbung und fluoreszenzbasierten Charakterisierung eukariotischer Zellen [9].

Im ersten Schritt wurden die Zellkulturkammern mit integrierter Membran mit mittels Celltracker Green CMFDA (Invitrogen) vital gefärbten Saccharomyces cerevisiae Zellen bestückt. Nach anschließendem Auffüllen der Kreisläufe mit Medium folgten der Betrieb der Pumpen mit einem Volumenstrom von $175 \mu \mathrm{l} / \mathrm{min}$ sowie die kontinuierliche Erfassung der Fluoreszenz in 
den Zellkulturkammern ohne integrierte Membran. Dieses Signal entspricht der durch geschädigte Zellen freigesetzten Menge Farbstoff. Abschließend wurde in jeweils einen der beiden Kreisläufe Triton X-100 zugegeben. Im Vergleich zur Referenz zeigten die Kreisläufe mit Triton $X-100$ einen signifikanten Anstieg der Fluoreszenz, siehe Abbildung 7.

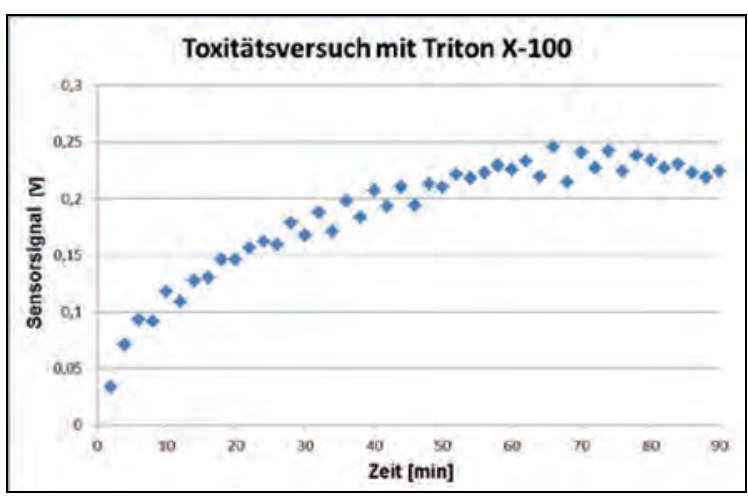

Abb. 7. Zeitlicher Verlauf des referenzierten Fluoreszenzsignals.

\subsection{Integrierte Reflexionsschichten}

Die an der Hochschule Mittweida entwickelte Kombination aus Pulsed Laser Deposition (PLD) und Laserpulsspannungsrelaxation (LSR) ermöglicht die Herstellung extrem harter, spannungsfreier, haftfester und biokompatibler Schichten aus tetraedrisch gebundenem amorphen Kohlenstoff (ta-C) [10, 11].

Mit dem Ziel, die Sensitivität der Online Fluoreszenz-Überwachung zu verbessern, erfolgte eine Analyse der Eignung dieser ta-CSchichten als Reflektoren. Dazu wurden etablierte Deckel zum Verschließen der Zellkulturkammern (Stahl) an der zur Mikrofluidik zeigenden Seite, siehe Abbildung 3 , mit ta-C beschichtet und anschließend charakterisiert.

Mit dem Fluoreszenzmesssystem erfolgte die Bestimmung der Eigenfluoreszenz. Die untersuchten, unterschiedlich dicken ta-C-Schichten zeigten in allen Fällen eine niedrige Eigenfluoreszenz, die mit denen von Gold oder Silizium vergleichbar ist. Daraus resultiert, dass durch die Beschichtung eines Werkstoffes mit ta-C, die Eigenfluoreszenz des Grundwerkstoffs abgeschirmt werden kann.

Mit dem Spektrometer F20 der Firma Filmetrics konnte die absolute, wellenlängenabhängige Reflexion der ta-C-Schichten auf Stahlsubstraten im Bereich $380 \mathrm{~nm}$ bis $1035 \mathrm{~nm}$ gemessen werden. Wie in Abbildung 8 ersichtlich, entstehen durch Interferenz schichtdickenabhängige Reflexionsmaxima und -minima.

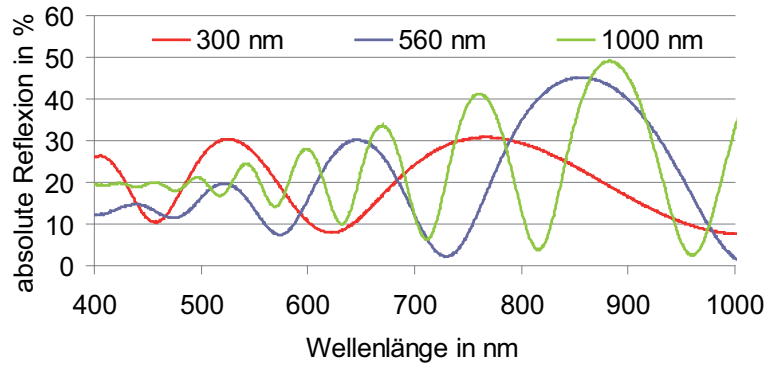

Abb. 8. Absolute Reflexion in Abhängigkeit von Schichtdicke und Wellenlänge.

Je nach abgeschiedener ta-C-Schichtdicke kann z. B. für eine bestimmte Wellenlänge definiert ein Reflexionsmaximum erzeugt werden.

Auf Basis der Reflektionsfunktionen wurden geeignete Schicht-Farbstoff-Kombinationen ermittelt. So eignet sich beispielsweise eine ta-C-Schicht mit einer Dicke von 1000 nm für eine Sensitivitätssteigerung beim Nachweis der Fluoreszenzmarker Cy7 (BD Biosciences) oder QDOT800 (Nanocrystal). Die Anregungswellenlänge liegt bei diesen Farbstoffen im Bereich eines Reflexionsminimums und die Emissionswellenlänge im Bereich eines Reflektionsmaximums, siehe Abbildung 9.

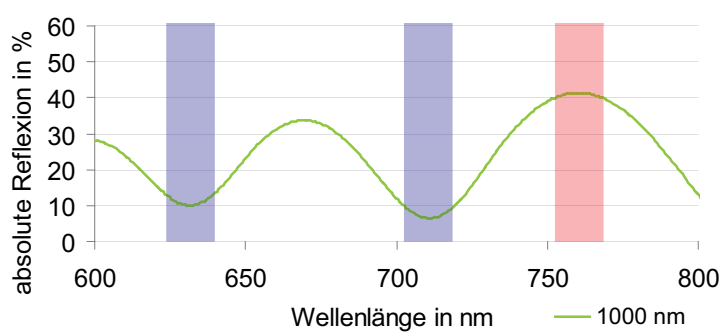

Abb. 9. Absolute Reflexion in Abhängigkeit der Wellenlänge für $1000 \mathrm{~nm}$ dicke ta-C-Schicht sowie Wellenlängenbereiche für Anregung (blau) und Emission (rot).

Um eine Erhöhung der absoluten Reflexion der ta-C-Schichten zu erreichen, werden in weiterführenden Untersuchungen Multilagen-Schichtsysteme aus ta- $C$ in Kombination mit Metallen hergestellt und analysiert.

\section{Zusammenfassung und Ausblick}

Eine geschlossene Technologiekette für die schnelle, flexible und preiswerte Herstellung von Lab-on-a-Chip-Systemen als Multilagenaufbau lasermikrostrukturierter Folien konnte erfolgreich etabliert werden.

Aufbauend auf dynamischen Multi-Organ-Chips für die Substanztestung an langzeitkultivierten humanen 3D-Co-Kulturen erfolgte die Entwicklung eines Multilagen-Demonstrator-Systems sowie die erfolgreiche Demonstration dessen 
Funktionalität am Beispiel eines Substanztestungs-Assays.

In weiterführenden Arbeiten soll die Multilagentechnologie optimiert und mit ihrer Hilfe weitere anwendungsspezifischer Lab-on-aChip-Systeme entwickelt und charakterisiert werden.

Erste Untersuchungen eines Ansatzes zur Steigerung der Sensitivität der Online Fluoreszenz-Überwachung durch Integration funktionaler Reflexionsschichten zeigen die Eignung von mittels PLD und LSR hergestellten ta-C-Schichten als Reflektor.

In zukünftigen Arbeiten soll das Potential von Reflektoren auf Basis von ta-C-MetallMultilagen-Schichtsystemen charakterisiert werden. Als Ziel steht dabei die Minimierung bzw. Maximierung der prozentualen absoluten Reflexion der Anregungswellenlänge bzw. Emissionswellenlänge.

\section{Danksagung}

Die Autoren danken dem Freistaat Sachsen und der Europäischen Union (SAB Projekt UNILOC) sowie dem BMWI (ZIM Projekt RoVitaS) für die finanzielle Unterstützung.

Die Autoren danken der GeSiM mbH für die Unterstützung bei der Softwareentwicklung für das Zwei-Ebenen-Portal-Robotik-System.

\section{Referenzen}

[1] U. Marx, H. Walles, S. Hoffmann, G. Lindner, R. Horland, F. Sonntag, U. Klotzbach, D. Sakharov, A. Tonevitsky und R. Lauster: 'Human-on-a-chip' Developments: A Translational Cuttingedge Alternative to Systemic Safety Assessment and Efficiency Evaluation of Substances in Laboratory Animals and Man? In: at - ATLA (2012) Nr. 40, S. 235-257.

[2] U. Marx et a.: Developing a human chip-based platform for repeated dose toxicity testing. In: at AXLR8 Alternative Testing Strategies Progress Report (2012), S. 210-218.

[3] Wagner, I. et al.: A dynamic multi-organ-chip for long-term cultivation and substance testing proven by $3 \mathrm{D}$ human liver and skin tissue coculture, Lab on a Chip 13, $3538-3547,2013$, DOI: 10.1039/C3LC50234A.

[4] Baker, M.: A LIVING SYSTEM ON A CHIP, Nature 471, 661-665, 2011.

[5] F. Schmieder et al.: Automated universal chip platform for fluorescence based cellular assays. In: at - Biomedical Engineering/ Biomedizinische Technik (2012), S. 340-343.
[6] F. Sonntag et al.: Universelle Plattform für die automatisierte, amperometrische Überwachung von Reportergenassays. In: Dresdner Beiträge Medizintechnik (2012), S. 189-192, ISBN: 978-3942710-96-1.

[7] F. Sonntag et al.: Universelle Geräteplattform für das automatisierte Handling zellbasierter Assays. In: Dresdner Beiträge Sensortechnik (2011). ISBN: 9783942710534

[8] M. Busek et al.: Peristaltic pneumatic pump characterisation via $\mu \mathrm{PIV}$, non-invasive pressure measurement and simulation. In: Journal of Sensors and Sensor Systems 2013, akzeptiert

[9] M. Ruhnow et al.: Universelle Plattform für die automatisierte Vitalfärbung und fluoreszenzbasierte Charakterisierung eukariotischer Zellen. In: Dresdner Beiträge Medizintechnik (2012), S. 193-196, ISBN 978-3-942710-96-1.

[10] K. Günther et al.: Tribological properties of superhard amorphous carbon (TA-C) Layers microstructured by FS-Laserpulses. In: Surface Modification Technologies XXIV, Vol. 24, ISBN: 978-81-910571-2-6

[11] A.-C. Teichmann et al.: Effect of microstructured and ta-C coated glass-surfaces on cell growth. In: NWK 12, 14.04.2011 - Wernigerode/Deutschland, S. $35-39$, ISBN: 978-3-00-034329-2 